\title{
Ion/Neutral, Ion/Electron, Ion/Photon, and Ion/lon Interactions in Tandem Mass Spectrometry: Do We Need Them All? Are They Enough?
}

\author{
Scott A. McLuckey, Marija Mentinova \\ Department of Chemistry, Purdue University, West Lafayette, IN 47907-2084, USA
}

\begin{abstract}
A range of strategies and tools have been developed to facilitate the determination of primary structures of analyte molecules of interest via tandem mass spectrometry (MS/MS). The two main factors that determine the primary structural information present in an MS/MS spectrum are the type of ion generated from the analyte molecule and the dissociation method. The ion type subjected to dissociation is determined by the ionization method/conditions and ion transformation processes that might take place after initial gas-phase ion formation. Furthermore, the range of analyte-related ion types can be expanded via derivatization reactions prior to mass spectrometry. Dissociation methods include those that simply alter the population of internal states of the mass-selected ion (i.e., activation methods like collision-induced dissociation) as well as processes that rely on the transformation of the ion type prior to dissociation (e.g., electron capture dissociation). A variety of ion interactions have been studied for the purpose of ion dissociation and ion transformation, including ion/neutral, ion/photon, ion/electron, and ion/ ion interactions. A wide range of phenomena have been observed, many of which have been explored/developed as means for structural analysis. The techniques arising from these phenomena are discussed within the context of the elements of structural determination in tandem mass spectrometry: ion-type definition and dissociation. Unique aspects of the various ion interactions are emphasized along with any barriers to widespread implementation.
\end{abstract}

Key words: Tandem mass spectrometry, Dissociation methods, Activation methods

\section{Introduction}

$\mathrm{G}$ as-phase reactions have long played important roles in mass spectrometry, both for the purpose of ionization and - in the case of molecular mass spectrometry - structural characterization. Emphasis is placed here on the primary structural characterization of molecules of interest (i.e., bond connectivity) via their gas-phase ionic surrogates. When reactions take place within the context of tandem mass spectrometry (i.e., between stages of mass analysis), precursor-product relationships can be clearly determined, and a significant reduction in chemical noise can be achieved [1]. The value of a tandem mass spectrometry experiment,

Correspondence to: Scott A. McLuckey; e-mail: mcluckey@purdue.edu however, is also clearly linked to the quality and quantity of information that is forthcoming from the reaction. For this reason, novel reactions have been explored and developed since the earliest days of analytical tandem mass spectrometry. We have seen reactions in tandem mass spectrometry expand from the unimolecular dissociation of metastable ions and ions activated by keV energy collisions to processes that involve the interactions of ions with surfaces, photons, electrons, ions of opposite polarity, and with inert and reactive neutral species over a wide range of collision energies and time-scales [2].

Given the wide range of interactions that can now be effected in tandem mass spectrometry, it is useful to place them all into the broad context of structural characterization in mass spectrometry. The discussion begins with a few general considerations for structural characterization by tandem mass spectrometry that include options before 
mass-selected ions are subjected to a reaction as well as options for processes that take place between the stages of MS/MS. With these in mind, it is then possible to discuss each type of interaction from a common standpoint. Collision-induced dissociation (CID), which falls into the ion/neutral interaction category, is regarded as the "default" process in MS/MS. It is the technique against which all others must be compared for reasons discussed below. For any other approach to find broad use, it must offer a compelling advantage over CID in a widely performed measurement. Each interaction is therefore discussed very briefly, with emphasis placed on general considerations and features that might make it attractive relative to CID, while current barriers to widespread implementation are also mentioned.

\section{Structural Characterization via MS/MS}

The identification and structural characterization of compounds in tandem mass spectrometry generally rely on the measurement of the mass of the precursor ion, which is related to the molecule in a known fashion, such as the molecular ion $\left(\mathrm{M}^{+}\right)$, the protonated molecule $(\mathrm{M}+\mathrm{H})^{+}$, etc., and the fragmentation of the ion after it has been subjected to a dissociation method. It is also generally the case that the nature of the ion plays a major role in determining the favored dissociation channels. Most fragmentation processes are either charge-site or radical-site directed [3]. For this reason, the way in which charge is manifested in the precursor ion and the presence or absence of radical sites generally strongly influence fragmentation patterns and, as a result, the extent of structural information that is forthcoming from the MS/MS experiment. Hence, the first key decision in tandem mass spectrometry involves the selection of the ion type that is to be subjected to dissociation. This point is illustrated in Figure 1, which compares ion trap CID of the $(\mathrm{M}+2 \mathrm{H})^{2+},(\mathrm{M}-2 \mathrm{H})^{2-}$, and $(\mathrm{M}+\mathrm{Au}+\mathrm{H})^{2+}$ species of native somatostatin (AGCKNFFWKTFTSC with a disulfide linkage between the cysteine residues) with the electron transfer dissociation (ETD) of the $(\mathrm{M}+3 \mathrm{H})^{3+}$ ion. In the latter case, it is the $[\mathrm{M}+3 \mathrm{H}]^{2+\cdot}$ species that fragments. Each fragmenting ion

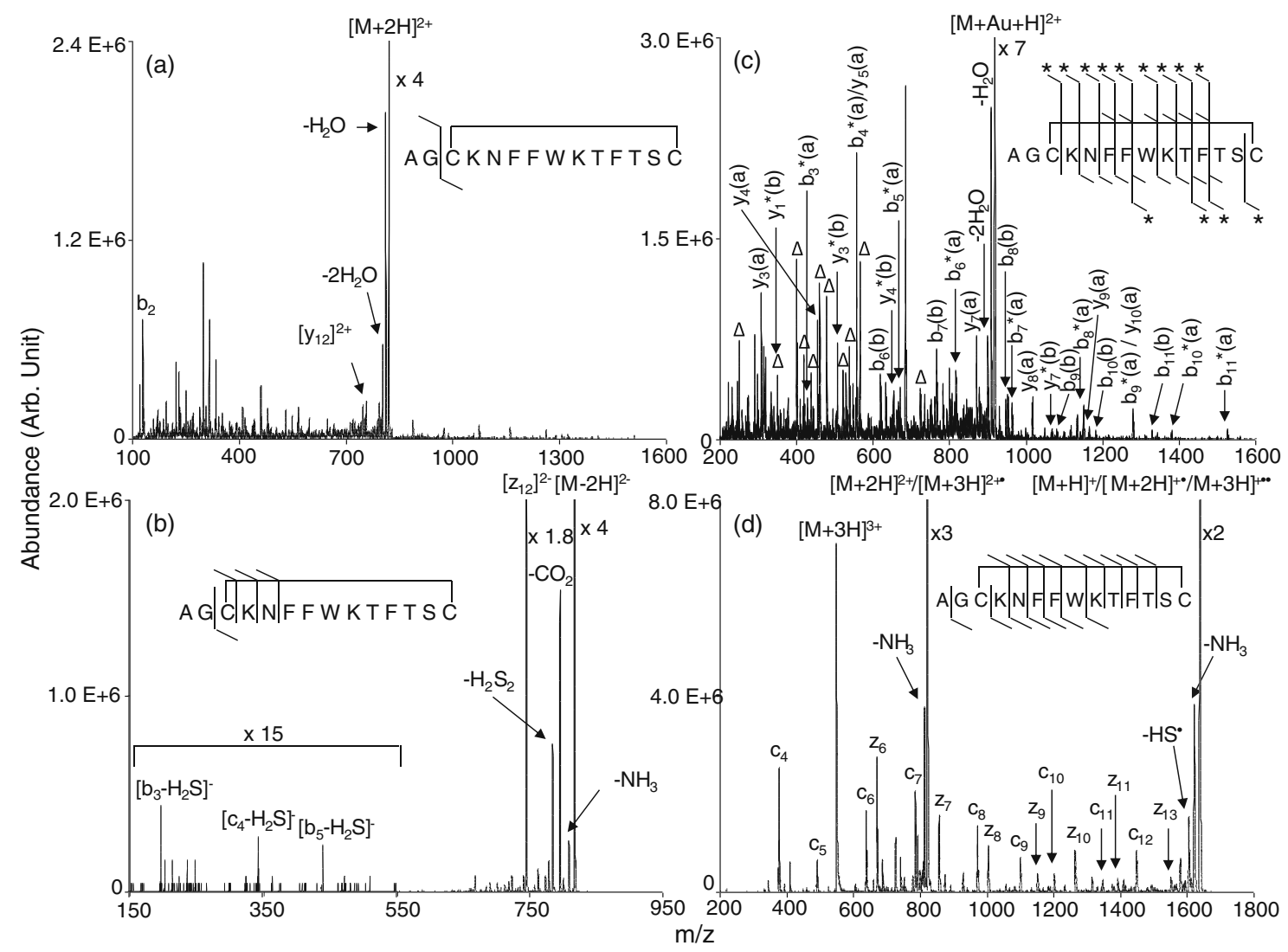

Figure 1. Product ion spectra from the dissociation of various doubly charged ion types of native somatostatin: (a) ion-trap CID of the $(\mathrm{M}+2 \mathrm{H})^{2+}$ ion, (b) ion-trap CID of the $(\mathrm{M}-2 \mathrm{H})^{2-}$ ion, $(\mathbf{c})$ ion-trap CID of the $(\mathrm{M}+\mathrm{Au}+\mathrm{H})^{2+}$ ion produced in the gas phase from $(\mathrm{M}+3 \mathrm{H})^{3+}$ via reaction with $\mathrm{AuCl}_{2}{ }^{-}$(the $\Delta$ symbol represents internal fragments from within the loop defined by the disulfide bridge that result from two consecutive amide bond cleavages, while the * symbol indicates the presence of $\mathrm{Au}^{+}$in the fragment, and (a) and (b) represent structures proposed in the original paper; see reference [6]), and (d) ETD of the $(\mathrm{M}+3 \mathrm{H})^{3+}$ ion (which leads to the dissociation of $(\mathrm{M}+3 \mathrm{H})^{2+}$ ) 
type exhibits a distinct product ion distribution, even though the fragmenting species carries the same absolute charge (i.e., all fragmenting ions are doubly charged). This example is hardly comprehensive with respect to the variety of doubly charged ions that might be derived from somatostatin. This particular set of ions was chosen because all of the data were collected using the same instrument platform, which facilitates direct comparison. Several options are available for defining the ion type for a given molecule of interest that is subjected to dissociation: (i) before ionization, the molecule of interest can be modified in a known manner to facilitate quantitation, ionization, and/or influence fragmentation [4, 5]; (ii) the ionization step itself offers many opportunities for defining the ion type via selection of the ionization method and conditions; and (iii), after ionization, several of the interaction types discussed here can be used to convert one ion type into another, as is the case in the $(\mathrm{M}+\mathrm{Au}+\mathrm{H})^{2+}$ experiment of Figure $1 \mathrm{c}$ and the ETD experiment of Figure 1d. That is, the structural information in Figure 1c and $\mathrm{d}$ was derived from ion types other than the initially selected triply protonated molecule, $(\mathrm{M}+3 \mathrm{H})^{3+}$.

The second major decision for structural characterization via MS/MS involves the selection of the dissociation method. Note that a distinction is made here between the term "dissociation method" and "activation method." The former term is broader in that it encompasses any process that leads to fragmentation. The process may or may not involve a change in ion type. In some dissociation processes, the ion type from which fragmentation takes place can differ from the massselected precursor ion. An example of a dissociation method that involves a change in the ion type is electron capture dissociation (ECD):

$$
(\mathrm{M}+\mathrm{nH})^{\mathrm{n}+}+\mathrm{e}^{-} \rightarrow(\mathrm{M}+\mathrm{nH})^{(\mathrm{n}-1)+* *} \rightarrow \text { fragments. }
$$

In this case, fragmentation takes place from a different ion type than the mass-selected precursor ion. The new ion type may also be formed with significant vibrational or electronic excitation that can drive subsequent dissociation. However, the key distinction here is that the fragmentation takes place from a new ion type. The term "activation method" refers to an approach that deposits energy into a precursor ion (i.e., alters the population of vibrational and/or electronic states) without involving a change in the ion type. The most common example is CID, which involves the conversion of relative translational energy in an ion/neutral target collision into internal modes of the collision partners:

$$
(\mathrm{M}+\mathrm{nH})^{\mathrm{n}+}+\mathrm{N}(\text { gas }) \rightarrow(\mathrm{M}+\mathrm{nH})^{\mathrm{n}+*}+\mathrm{N}^{*} \rightarrow \text { fragments }
$$

Other examples involve collisions with a surface (SID) and photoexcitation (e.g., IRMPD, UV-PD), provided they do not remove an electron. Electron detachment is an ion transformation process. The structural information that results from a dissociation method is therefore dependent upon ion type, how the internal states of the ion are populated, and the observation time-scale. In considering the characteristics of the various ion interaction types, it is therefore useful to consider them in terms of both energy transfer and transformation of ion type. Some approaches are restricted to energy transfer and do not change the ion type, while other ionic interactions can lead to ion-type transformation. Ion-type transformation may lead to more or less "spontaneous" dissociation, as in ECD, or it may be used in conjunction with an activation method. Figure 1c provides an example of the latter case where the $(\mathrm{M}+3 \mathrm{H})^{3+}$ ion was transformed into the $(\mathrm{M}+\mathrm{Au}+\mathrm{H})^{2+}$ ion in the gas phase via reaction with $\mathrm{AuCl}_{2}^{-}$[6] and subsequently probed via iontrap CID. Figure 2 provides a schematic picture of choices available for ion type and dissociation method, and Table 1 summarizes the elements that can go into an overall strategy for structural characterization, listing a few options within each element for illustration. The possible steps include molecule modification, ion generation method, ion-type transformation method, and dissociation method. Ionization and dissociation are required elements, whereas derivatization prior to ionization and ion-type transformation after ionization are elements that can be incorporated if needed. Table 2 summarizes the ion activation techniques (no change in ion type), ion dissociation techniques that rely on a change in ion type, and ion transformation techniques (no dissociation) according to the interaction types discussed further below.

The structural information that can ultimately be obtained from a tandem mass spectrum is the primary criterion in assessing an approach. However, there are also important practical considerations for analysis, such as efficiency and speed. Efficiency can be defined here as the fraction of massselected precursor ions that are converted to informative products. Clearly, a process of low efficiency will be limited in applicability to highly abundant precursor ions. The timescale over which the process takes place is important in determining its compatibility with respect to implementation with on-line separations. Other practical considerations include the ease and cost of implementation of a method. The instrument platform usually places constraints on the range of processes and conditions that can be accessed. For example, ions typically travel at $\mathrm{keV}$ translational energies between stages of MS in sector and tandem TOF instruments. Therefore, processes that involve low translational energy ions, such as ion/molecule reactions, are difficult to implement between stages of MS in such instruments. Furthermore, the choice of ionization method is generally limited for any particular instrument.

The remaining discussion provides brief commentary on the various methods that fall into each of the interaction type categories within the context provided above. No attempt is made to review the various interactions and methods. 


\section{Structural Information $=\mathrm{f}$ (ion-type, dissociation method)}

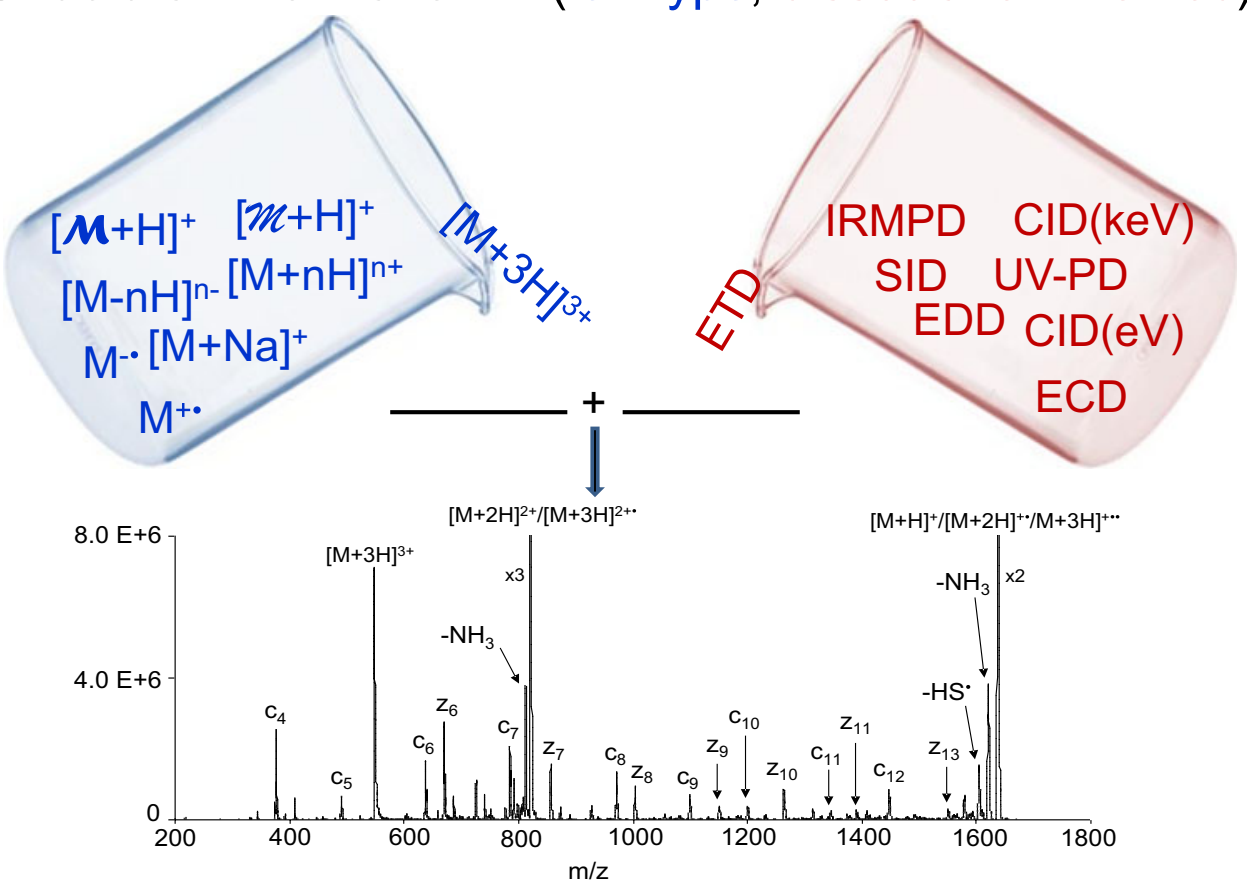

Figure 2. Schematic picture depicting selection of ion-type and dissociation method for structural characterization in tandem mass spectrometry. Note that some dissociation methods may not be compatible with all ion types. $\mathrm{M}$ represents a molecule of interest. $\mathbf{M}$ and $M$ represent intentionally modified forms of $\mathbf{M}$. Modifications might be made for one or more reasons, such as to facilitate quantitation, ionization, and/or fragmentation

Table 1. A summary of the steps that can be involved in ion-type generation and dissociation in tandem mass spectrometry. Ion-type generation involves the possible use of wet chemistry to derivatize the analyte, the selection of an ionization method, and possible gas-phase ion transformation. Ion dissociation in MS/MS generally follows the formation of an initial ion type. It may involve the application of an activation method, such as CID or IRMPD, to the initially formed ion type, or it may involve an ion transformation step that leads to the dissociation of a different ion type, as occurs with ECD or EDD. The latter are ion dissociation methods but are not strictly ion activation methods, as defined here, because they involve an ion transformation that fundamentally alters the ion chemistry

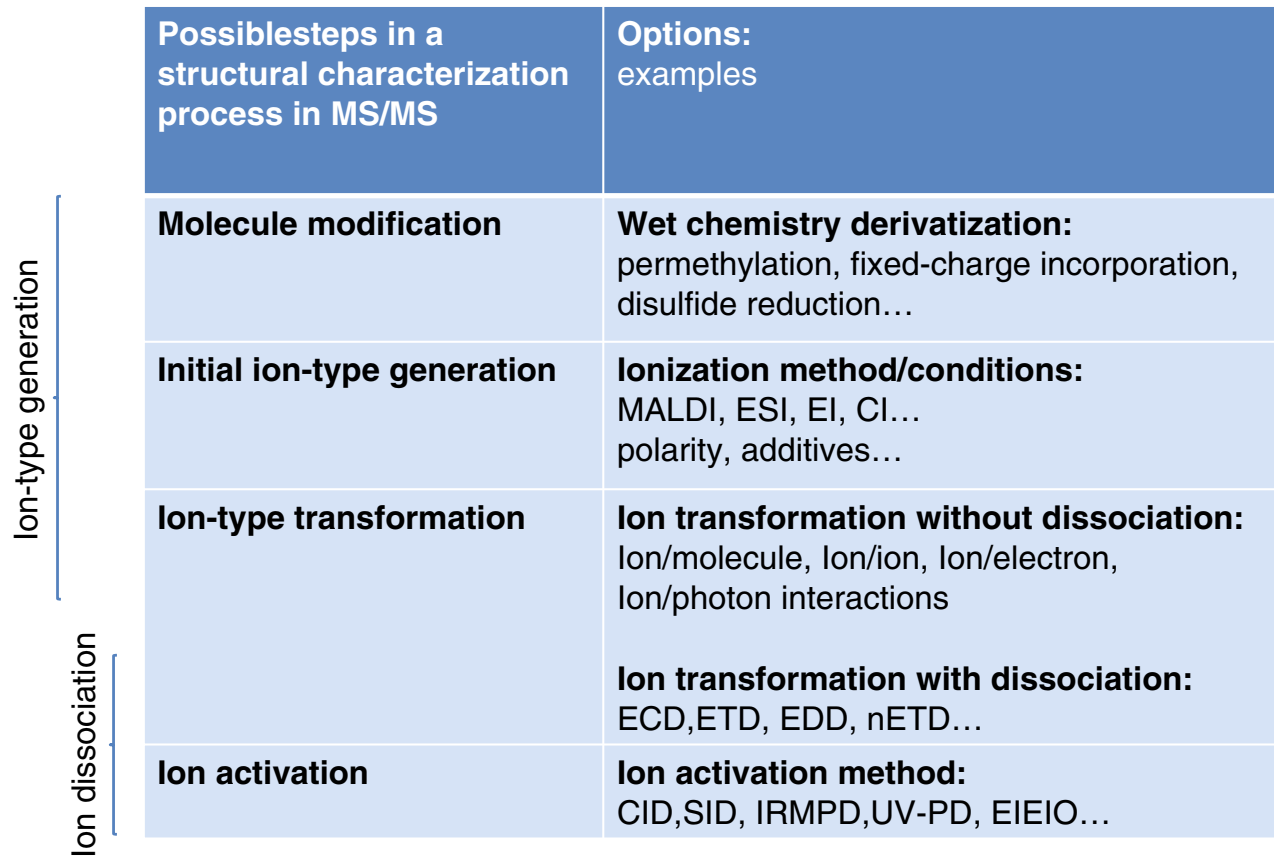


Table 2. Summary of ion activation, ion dissociation, and ion transformation approaches according to ion interaction type ( $\Delta=$ "change in")

\begin{tabular}{|c|c|c|c|}
\hline & $\begin{array}{l}\text { Ion activation } \\
\text { No } \Delta \text { ion type }\end{array}$ & $\begin{array}{l}\text { Ion dissociation } \\
\text { No } \Delta \text { ion type }\end{array}$ & $\begin{array}{c}\Delta \text { Ion type } \\
\text { No dissociation }\end{array}$ \\
\hline Ion/neutral (ion/gas or surface) & CID, SID, MAD & MAD/MIDI ECID & $\begin{array}{l}\mathrm{I} / \mathrm{M} \mathrm{H}^{+}, \mathrm{e}^{-} \text {transfer, } \mathrm{H} / \mathrm{D} \text { exchange } \\
\text { Collisional ionization, etc. }\end{array}$ \\
\hline Ion/photon & UV-PD, IRMPD & & $\mathrm{e}^{-}$photodetachment \\
\hline Ion/electron & EIEIO, EED & $\begin{array}{l}\text { ECD, HECD, Al-ECD, } \\
\text { EDD, El+dissociation }\end{array}$ & $\begin{array}{l}\mathrm{e}^{-} \text {capture } \\
\mathrm{e}^{-} \text {detachment (anions) } \\
\mathrm{e}^{-} \text {ionization (cations) }\end{array}$ \\
\hline Ion/ion & & ETD, nETD & $\begin{array}{l}\mathrm{I} / \mathrm{I} \mathrm{H}^{+} \text {or } \mathrm{e}^{-} \text {transfer, Heavy ion transfer, } \\
\text { Charge inversion, Covalent modification, etc. }\end{array}$ \\
\hline
\end{tabular}

Rather, emphasis is placed on unique features of the various interaction types.

\section{Ion/Neutral Interactions}

This broad category includes several types of ion/neutral collisions. Those relevant to tandem mass spectrometry are energetic collisions between ions and ground-state neutral species (e.g., ion/neutral collision-induced dissociation and collisional electron detachment reactions), lowenergy reactive collisions (i.e., ion/molecule reactions), collisions of ions with neutral species in excited electronic states (e.g., metastable atoms), and collisions of ions with a surface (e.g., surface-induced dissociation). (Note that elastic ion/neutral collisions also play a major role in ion mobility measurements, but this topic falls outside the present scope.)

Collision-Induced Dissociation Much has already been written about CID (reaction 2) and the wide range of conditions under which it can be implemented [7-9]. It is a true activation method in that it transfers energy into the precursor ion without changing its ion-type. While no tandem mass spectrometer can readily access the entire range of conditions that can give rise to CID, essentially all tandem mass spectrometers are capable of implementing CID under some conditions. If a structural characterization problem can be addressed via CID, this will generally be the preferred approach, because virtually all tandem mass spectrometers are designed around the implementation of ion/neutral gas collisions. Important characteristics of CID are its universal nature, its ease of implementation, its high efficiency (at least under some conditions), and its speed, particularly in beam-type instruments.

Ion/Neutral Collisional Ionization (Charge Permutation) At $\mathrm{keV}$ collision energies, endothermic charge permutation reactions [10] can occur, such as charge stripping (reaction 3 represents charge stripping from a radical cation), charge inversion (reaction 4 represents charge inversion of a radical anion), electron transfer (for singly charged ions, this constitutes the first step in a neutralization/reionization experiment; see reaction 5 [11]) and collisional ionization (reaction 6; e.g., the second step in a neutralization/ reionization experiment). Electron transfer to fast doubly charged cations has also been reported, and can give rise to fragmentation in a process termed electron-capture-induced dissociation [12] (ECID, reaction 7):

$$
\begin{aligned}
& \text { fast } \mathrm{M}^{+\cdot}+\mathrm{N} \rightarrow \mathrm{M}^{2+}+\mathrm{N}+\mathrm{e}^{-} \\
& \text {fast } \mathrm{M}^{-\cdot}+\mathrm{N} \rightarrow \mathrm{M}^{+\cdot}+\mathrm{N}+2 \mathrm{e}^{-}
\end{aligned}
$$

$$
\text { fast } \mathrm{M}^{+\cdot}+\mathrm{N} \rightarrow \mathrm{M}+\mathrm{N}^{+\cdot}
$$

$$
\text { fast } \mathrm{M}+\mathrm{N} \rightarrow \mathrm{M}^{+\cdot}+\mathrm{N}+\mathrm{e}^{-}
$$

$$
\text { fast } \mathrm{M}^{2+}+\mathrm{N} \rightarrow \mathrm{M}^{+\cdot *}+\mathrm{N}^{+\cdot} \rightarrow \text { fragments. }
$$

Reactions 3-7) are ion transformation processes that may or may not result in fragmentation. Such processes are not important at low collision energies. Even at high collision energies for polyatomic ions, collision-induced dissociation often dominates. Collisional ionization processes have not found much use in biological tandem mass spectrometry, in part due to the paucity of instruments that employ $\mathrm{keV}$ collision energies and the relatively low velocities of high mass ions, which result in small cross-sections for collisional electron detachment.

Ion/Molecule Reactions The study of reactive ion/molecule collisions at thermal and near-thermal energies has a long history in mass spectrometry. The use of ion/molecule reactions in tandem mass spectrometry has included, for example, the examination of H/D exchange [13], HI attachment [14], proton transfer [15], and functional-groupspecific covalent reactions [16]. Reaction 8 represents proton transfer from a multiply protonated species:

$$
(\mathrm{M}+\mathrm{nH})^{\mathrm{n}+}+\mathrm{B} \rightarrow(\mathrm{M}+(\mathrm{n}-1) \mathrm{H})^{(\mathrm{n}-1)+}+\mathrm{BH}^{+} .
$$


Thermal energy ion/molecule reactions involving groundstate reactants are not used as ion activation or ion dissociation methods. Rather, they play an important role in ion transformation applications (e.g., charge state manipulation), and are therefore sometimes used in conjunction with an activation method (e.g., CID). Ion/molecule reactions can be quite efficient (e.g., $100 \%$ of the analyte ions can undergo reaction in favorable cases), depending upon the magnitude of the rate constant, the number density of the reagent that can be generated, and the time available for reaction. The range of reactions that can be accessed is constrained by the ability to generate an adequate number density of the neutral reagent. For this reason, neutral reagents are typically limited to relatively small species with relatively high vapor pressures, although desorption methods, such as laser-induced acoustic desorption (LIAD) [17], can expand the range of neutral analytes available for consideration.

Ion/Metastable Atom Interactions Recent reports have described the interaction of analyte ions with atoms in excited electronic states as a means for ion dissociation [18]. Such atoms may either have low translational energies [19] or high translational energies [20]. This experiment has been coined metastable atom-induced dissociation (MAD) and metastable-induced dissociation of ions (MIDI). Several mechanisms that lead to dissociation have been identified, depending upon conditions and the analyte ion charge state and polarity. The transfer of an electron from a metastable atom to a multiply charged analyte constitutes a dissociation process that involves ion transformation, and leads to products similar to those generated by electron capture and electron transfer from anions:

$$
(\mathrm{M}+\mathrm{nH})^{\mathrm{n}+}+\mathrm{A}^{*} \rightarrow(\mathrm{M}+\mathrm{nH})^{(\mathrm{n}-1)+\cdot *}+\mathrm{A}^{+\cdot} \rightarrow \text { fragments. }
$$

A Penning ionization process that gives rise to dissociation products has also been described for cations, which provides a mechanism for the dissociation of singly charged cations [21]:

$(\mathrm{M}+\mathrm{nH})^{\mathrm{n}+}+\mathrm{A}^{*} \rightarrow(\mathrm{M}+\mathrm{nH})^{(\mathrm{n}+1)+* *}+\mathrm{A}+\mathrm{e}^{-} \rightarrow$ fragments,

and for anions:

$(\mathrm{M}-\mathrm{nH})^{\mathrm{n}-}+\mathrm{A}^{*} \rightarrow(\mathrm{M}-\mathrm{nH})^{(\mathrm{n}-1)-* *}+\mathrm{A}+\mathrm{e}^{-} \rightarrow$ fragments.

When the metastable atoms have relatively high translational energies, CID can contribute. The electronic excitation of an analyte ion (without electron transfer or detachment) with the concomitant relaxation of the atom- which would qualify as an activation process - is also possible, but the extent to which such a process contributes is unclear.

MAD/MIDI is relatively new and has been applied to a very limited number of analyte classes, making its ultimate potential as a dissociation method difficult to assess. Reported efficiencies $(<5 \%)$ probably do not reflect those from a highly optimized system with a bright metastable atom source. Some capabilities that complement CID, such as preservation of post-translational modifications, overlap with those of other methods (e.g., ETD, ECD) but the applicability of MAD to singly charged cations is potentially important. The requirement of a metastable atom source represents a barrier to widespread implementation.

Ion/Surface Interactions This category includes the softlanding experiment [22] in which a mass spectrometer is used to purify a species for deposition onto a surface and energetic ion/surface collisions for ion activation. The latter application, referred to as surface-induced dissociation (SID) [23-25], is a variation of CID.

$$
\begin{aligned}
(\mathrm{M}+\mathrm{nH})^{\mathrm{n}+}+\mathrm{N}(\text { surface }) & \rightarrow(\mathrm{M}+\mathrm{nH})^{\mathrm{n}+*}+\mathrm{N}^{*} \\
& \rightarrow \text { fragments }
\end{aligned}
$$

The surface provides for high center-of-mass collision energies and relatively short activation times. The energy transfer distribution has been shown to generally be narrower than those provided under most CID conditions and more precisely variable. The relatively fast activation has been shown to give rise to symmetrical charge state distributions from the dissociation of dimeric and multimeric complexes, whereas most CID studies have shown asymmetric partitioning of charge [26]. SID, therefore, appears to have advantages over CID for the examination of large biomolecule complex ions. SID has largely been implemented with specially constructed instrumentation, and has not seen widespread adoption. If SID could be implemented in commercial instrumentation without compromising CID or adding substantially to the cost, this approach could see wider use. Developments in this direction are underway [26].

\section{Ion/Photon Interactions}

Ion spectroscopy has been actively pursued for many years, with significant advances made in bio-ion spectroscopy in recent years [27]. Ion/photon interactions are particularly attractive from the standpoint of the potential to obtain wavelength-dependent information and the practical advantage of requiring no gases to be admitted into the vacuum system. While CID is largely "universal" or "nonspecific," the use of photons as a means for ion excitation promises to 
add levels of specificity via the dependence of absorbance upon wavelength, which cannot be provided by the other types of interactions. Given the impracticality of measuring the absorbance spectrum of an ion directly, fragmentation is used as a signature of photon absorption. The ability to generate overall dissociation yields and fragmentation patterns as a function of wavelength is very attractive. Furthermore, selective activation via chromophore-specific irradiation is also attractive. However, issues remain with regard to efficiency, speed, ease of implementation, and cost, which must be addressed before the full potential of ion/ photon interactions can be realized for ion structure characterization.

To date, the major applications of photodissociation in analytical MS have been infrared multiphoton dissociation (IRMPD) [28-30] (reaction 13 for a protonated molecule) and ultraviolet photodissociation (UV-PD) $[31,32]$, the latter of which is usually a single-photon process $(\mathrm{n}=1)$.

$$
(\mathrm{M}+\mathrm{H})^{+}+\mathrm{nh} v \rightarrow(\mathrm{M}+\mathrm{H})^{+*} \rightarrow \text { fragments }
$$

IRMPD, usually implemented with a continuous-wave fixed-wavelength IR laser $(10.6 \mu \mathrm{m})$, shares commonalities with some forms of CID [33], as it is a slow heating method. It has proven to be particularly useful when the presence of a collision-gas is deleterious to MS performance, as in FTICR, and has recently been coupled with derivatization approaches to provide a degree of selectivity in ion activation [34]. Black-body infrared dissociation (BIRD) [35] is another IR multiphoton approach, typically used in FT-ICR instruments, that relies on the absorption of blackbody radiation from the environment. Rather than being used as a structural tool, this broadband activation method is primarily used as a tool to generate information about dissociation dynamics via the measurement of fragmentation kinetics at well-defined temperatures. UV-PD techniques at several fixed wavelengths have been examined. Of particular note for peptides are the unique fragmentation reactions observed for singly protonated peptides using $157 \mathrm{~nm}$ photons [36] that enable de novo sequencing. Single-photon UV-PD is particularly well suited to tandem time-of-flight instruments, for which reaction conditions between mass analysis stages are often limited to relatively short interaction times.

While IRMPD and UV-PD are ion activation methods, photodetachment can be used as an ion transformation technique. Although, in principle, it is possible to photoionize a cation, the high ionization energies of cations makes such a prospect technologically challenging (e.g., vacuum UV irradiation would be needed for single-photon photoionization). Photodetachment of electrons from multiply charged anions, however, requires lower energies and has been demonstrated [37] (reaction 14). Dissociation of ions following photodetachment has also been described [38].
Hence, limited ion-type transformation is possible with light, which can also constitute a dissociation method if fragmentation results.

$$
(\mathrm{M}-\mathrm{nH})^{\mathrm{n}-}+\mathrm{h} v \rightarrow(\mathrm{M}-\mathrm{nH})^{(\mathrm{n}-1)-\cdot}+\mathrm{e}^{-}
$$

Ion/light interactions are well established in tandem mass spectrometry, and their importance will likely grow as technology advances. The need for a light source (with BIRD constituting an exception) introduces a cost/implementation barrier. However, the variable wavelength provides degrees of specificity and selectivity that are unique among the interaction types. For this reason, we can expect to see further exploration of wavelength dependence and combinations of ion transformation with photoactivation techniques.

\section{Ion/Electron Interactions}

The first work utilizing the electron irradiation of ions within the context of tandem mass spectrometry was carried out by Freiser et al. using a technique referred to as "electroninduced excitation of ions from organics" (EIEIO) [39], whereby electrons were examined as an alternative to collisions for ion activation in MS/MS.

$$
\begin{aligned}
(\mathrm{M}+\mathrm{H})^{+}+\mathrm{e}^{-}(\text {low eV }) & \rightarrow(\mathrm{M}+\mathrm{H})^{+*}+\mathrm{e}^{-} \\
& \rightarrow \text { fragments }
\end{aligned}
$$

Relatively little extension of the use of electrons beyond EIEIO in MS/MS took place until the discovery of ECD of multiply charged ions [40] (reaction 1). ECD proved, in many cases, to provide more extensive primary sequence information from multiply protonated peptides and proteins than CID, because CID typically occurs by charge-directed pathways that are sequence and charge-state dependent, and, importantly, preserved labile post-translational modifications so that they could be localized. This addressed a serious shortcoming of CID for a widely shared analytical problem. Subsequent work demonstrated variations of the ECD experiment in which collisional or IR activation of ions prior to (i.e., activated ion ECD, AI-ECD [41]) or after electron capture was carried out, and in which electron energies were increased (i.e., hot ECD or HECD [42]), resulting in greater dissociation yields.

Since the early ECD work, it has been demonstrated that electron energy is an important variable for generating a variety of ion-type transformations [43]. Electron detachment from either cations (singly or multiply charged), which has been termed "tandem ionization" [44], or multiply charged anions has been demonstrated. Fragmentation has been noted from electron detachment using relatively highenergy electrons. In the case of electron detachment from 
multiply charged anions, the process has been coined "electron detachment dissociation" (EDD) [45] (reaction 16).

$$
\begin{aligned}
(\mathrm{M}-\mathrm{nH})^{\mathrm{n}-}+\text { fast } \mathrm{e}^{-} & \rightarrow(\mathrm{M}-\mathrm{nH})^{(\mathrm{n}-1)-\cdot *}+2 \mathrm{e}^{-} \\
& \rightarrow \text { fragments }
\end{aligned}
$$

In the case of electron detachment from cations, some of the initially formed cations of higher charge have apparently undergone a subsequent electron capture event followed by dissociation (reaction 17).

$$
\begin{aligned}
(\mathrm{M}+\mathrm{H})^{+}+\text {fast } \mathrm{e}^{-} & \rightarrow(\mathrm{M}+\mathrm{H})^{2+\cdot}+\text { slow } \mathrm{e}^{-} \\
& \rightarrow(\mathrm{M}+\mathrm{H})^{+*} \rightarrow \text { fragments }
\end{aligned}
$$

The net result of the electron detachment/electron capture process is electronic excitation of the $(\mathrm{M}+\mathrm{H})^{+}$species, which has been termed electronic excitation dissociation (EED) [46]. Very recently, it was observed that electron capture by an anion can occur via the irradiation of anions with electrons of 4-5 eV [47]. These relatively new processes and the radical ions that are produced via electron detachment from even-electron multiply charged ions have not yet been studied extensively. However, the technologies developed to allow for the generation of high fluxes of electrons for ECD studies have enabled the study of these lower crosssection processes. Furthermore, interest in ion-electron interactions has stimulated the development of electrodynamic ion trap-based technology for their implementation with hybrid tandem mass spectrometers [48].

\section{Ion/Ion Reactions}

The ability to generate multiply charged ions has enabled the study and development of ion/ion reactions for analysis [4951]. High reaction efficiencies can be obtained due to the large cross-sections associated with ion/ion reactions and high reaction exothermicities [52]. Ion/ion reactions have proven to be remarkably versatile for ion transformation, as reagents have been identified that give rise to proton transfer $[53,54]$ :

$$
\begin{aligned}
& (\mathrm{M}+\mathrm{nH})^{\mathrm{n}+}+\mathrm{A}^{-} \rightarrow(\mathrm{M}+(\mathrm{n}-1) \mathrm{H})^{(\mathrm{n}-1)+}+\mathrm{AH} \\
& (\mathrm{M}-\mathrm{nH})^{\mathrm{n}-}+\mathrm{BH}^{+} \rightarrow(\mathrm{M}-(\mathrm{n}-1) \mathrm{H})^{(\mathrm{n}-1)-}+\mathrm{B},
\end{aligned}
$$

electron transfer $[55,56]$ :

$$
\begin{aligned}
(\mathrm{M}+\mathrm{nH})^{\mathrm{n}+}+\mathrm{A}^{-\cdot} & \rightarrow(\mathrm{M}+\mathrm{nH})^{(\mathrm{n}-1)+\cdot *}+\mathrm{A} \\
& \rightarrow \text { fragments } \\
(\mathrm{M}-\mathrm{nH})^{\mathrm{n}-}+\mathrm{B}^{+\cdot} & \rightarrow(\mathrm{M}-\mathrm{nH})^{(\mathrm{n}-1)-\cdot *}+\mathrm{B} \\
& \rightarrow \text { fragments, }
\end{aligned}
$$

metal ion transfer [57]:

$$
\begin{aligned}
& (\mathrm{M}+\mathrm{nH})^{\mathrm{n}+}+\mathrm{MetX}_{2}^{-} \\
& \quad \rightarrow(\mathrm{M}+\operatorname{Met}+(\mathrm{n}-2) \mathrm{H})^{(\mathrm{n}-1)+}+2 \mathrm{HX},
\end{aligned}
$$

complex formation [58]:

$$
\begin{aligned}
& (\mathrm{M}+\mathrm{nH})^{\mathrm{n}+}+(\mathrm{M}-\mathrm{xH})^{\mathrm{x}-} \\
& \quad \rightarrow(2 \mathrm{M}+(\mathrm{n}-\mathrm{x}) \mathrm{H})^{(\mathrm{n}-\mathrm{x})+},
\end{aligned}
$$

and selective covalent bond formation (e.g., Schiff base formation upon the reaction of primary amines in peptide ions with aldehyde-containing reagent anions [59, 60]). Reactions that involve multiple ion transfers in a single step have also been reported, and include those that give rise to charge inversion [61, 62], an example of which is depicted in reaction 24 :

$$
\begin{aligned}
& (\mathrm{M}-\mathrm{H})^{-}+(\mathrm{N}+\mathrm{nH})^{\mathrm{n}+} \rightarrow(\mathrm{M}+\mathrm{H})^{+} \\
& +(\mathrm{N}+(\mathrm{n}-2) \mathrm{H})^{(\mathrm{n}-2)+} .
\end{aligned}
$$

Ion/ion electron transfer to multiply charged cations can give rise to dissociation (i.e., electron transfer dissociation (ETD) [51], see reaction 20) that is generally analogous to that noted for ECD. Similarly, dissociation from ion/ion electron transfer from multiply charged anions [50], referred to as negative electron transfer dissociation [63] (nETD, see reaction 21), is similar to that noted in EDD. Hence, ETD and $\mathrm{nETD}$ constitute dissociation methods. Ion/ion reactions are commonly carried out in electrodynamic ion traps due to their ability to store ions simultaneously and in overlapping regions of space. Ion traps are common standalone instruments as well as components in many hybrid instruments. Hence, ETD and nETD can be implemented in many popular tandem MS platforms, provided the means for generating and delivering the reagent ions are in place. Commercially available instruments from several manufacturers support ion/ion electron transfer and proton transfer reactions, and these instruments are seeing growing use in proteomics applications. While there are many useful analytical applications for single-electron transfer and single-proton transfer, the range of chemistries potentially accessible via ion/ion reactions extends much further, making them perhaps the most flexible of the ion interactions for ion transformation.

\section{Discussion}

The preceding descriptions make clear that each of the ion interaction types has unique characteristics, as well as characteristics that overlap with other interaction types either for ion transformation or structural determination. Ion/ molecule, ion/electron, and ion/ion reactions are primarily 
methods for ion transformation that can take place in conjunction with fragmentation, as in ECD and ETD, or can be used in series with an activation method. Electron energy provides a useful variable in electron/ion interactions, but the processes are restricted to electron capture, electron detachment, or electronic excitation. Ion/molecule and ion/ ion reactions provide for a much more diverse array of chemical phenomenology, but each has unique challenges in generating and/or delivering the reagent species. Aside from differences in implementation, ion/molecule and ion/ion reactions show overlap in some reaction types but not in others, such as charge inversion, which is restricted to ion/ ion reactions, and metastable atom dissociation, which is an ion/neutral interaction process. As for pure activation methods, inelastic collision techniques (i.e., CID and SID) offer "universal" approaches, while the photon-based approaches offer the potential for greater selectivity than collision-based techniques.

Answers to the questions posed in the title can be addressed with analogy to a toolbox. As far as structural characterization is concerned, some of the interactions discussed here serve as tools for ion activation, some serve as tools for ion-type transformation, and some combine ion transformation with dissociation. Some tools are used often, while others are used only in very specialized circumstances. Some approaches have been or are being evaluated for their potential and may find no particular niche. Each of the general reaction types discussed here has unique characteristics and may therefore be uniquely well suited for a particular task. In this sense, the answer to the first question is "it would be nice if our toolbox was equipped with all of these techniques, but some tools are far more useful than others and we might not use some even if we could." It is impractical to design an instrument that allows for all of the interaction types. Hence, some of the techniques may never find extensive use in solving structural problems. Furthermore, while some may make important contributions in specific areas, others may never find an application for which its characteristics are sufficiently compelling to be adopted by anyone. Such is the way of technology. Hence, based on our current state of knowledge, it would be difficult to argue that we really do need all of the techniques that have been bestowed with acronyms.

As for the second question-whether current tools are enough - there will always be a need for new and improved tools if there are unmet needs. Even with all of the currently available options in derivatization, ionization, and dissociation, there are many unmet needs, as well as room for improvement in measurements that already provide useful information. These structural characterization needs are apparent at the boundaries of all of the application areas in modern molecular mass spectrometry (e.g., carbohydrates, lipids, high-mass biomolecules, large complexes, metabolomics, proteomics, etc.). Clearly, the answer to this question is "no." For this reason, it is important to continue to explore new reaction conditions and new reaction types, as well as to further develop some types of interactions that provide useful capabilities but may be lacking in, for example, efficiency, speed, or ease of implementation. Novel derivatization approaches, novel ionization methods or conditions, novel ion transformation, and new or improved ion activation approaches will be needed to address current and future challenges. While some of what has already been explored may not prove to be "needed" in the long run, we are certainly far from having everything we need.

\section{Acknowledgments}

The authors' work in the general area of structural characterization via mass spectrometry has been supported by the Office of Basic Energy Sciences, Division of Chemical Sciences under award no. DE-FG02-00ER15105. Instrument development and the application of ion/ion reactions to peptide and protein ions have been supported by the National Institutes of Health under grant GM 45372.

\section{References}

1. Busch, K.L., Glish, G.L., McLuckey, S.A.: Mass spectrometry/mass spectrometry: techniques and applications of tandem mass spectrometry. VCH Publishers, New York (1988)

2. Sleno, L., Volmer, D.A.: Ion activation methods for tandem mass spectrometry. J. Mass Spectrom. 39, 1091-1112 (2004)

3. McLafferty, F.W., Turecek, F.: Interpretation of Mass Spectra, 4th edn. University Science Books, Sausalito (1993)

4. Mendoza, V.L., Vachet, R.W.: Probing protein structure by amino acid specific covalent labeling and mass spectrometry. Mass Spectrom. Rev. 28, 785-815 (2009)

5. Froelich, J.M., Lu, Y., Reid, G.E.: In: March, R.E., Todd, J.F.J. (eds.) Chemical derivatization and multistage tandem mass spectrometry for protein structural characterization. Practical aspects of trapped ion mass spectrometry, vol. V, Chapter 4, pp. 83-119. CRC Press, Boca Raton (2010)

6. Mentinova, M., Han, H., McLuckey, S.A.: Dissociation of disulfideintact somatostatin ions: the roles of ion-type and dissociation method. Rapid Commun. Mass Spectrom. 23, 2647-2655 (2009)

7. McLuckey, S.A.: Collisional activation in analytical mass spectrometry. J. Am. Soc. Mass Spectrom. 3, 599-614 (1992)

8. Laskin, J., Futrell, J.H.: Activation of large ions in FT-ICR mass spectrometry. Mass Spectrom. Rev. 24, 135-167 (2005)

9. Wells, J.M., McLuckey, S.A.: Collision-induced dissociation (CID) of peptides and proteins. Meth. Enzymol. 402, 148-185 (2005)

10. He, M., McLuckey, S.A.: Charge permutation reactions in tandem mass spectrometry. J. Mass Spectrom. 39, 1231-1259 (2004)

11. Tureček, F.: Transient intermediates of chemical reactions by neutralization-reionization mass spectrometry. Top. Curr. Chem. 225, 77-129 (2003)

12. Vékey, K., Brenton, A.G., Beynon, J.H.: Electron capture-induced decomposition (ECID) - a new method to study electron capture collisions. Int. J. Mass Spectrom. Ion Processes 70, 277-300 (1986)

13. Green, M.K., Lebrilla, C.B.: Ion-molecule reactions and H/D exchange for structural characterization of biomolecules. In: Laskin, J., Lifshitz, C. (eds.) Principles of Mass Spectrometry Applied to Biomolecules, Chapter 3, pp. 119-145. Wiley, Hoboken (2006)

14. Stephenson Jr., J.L., McLuckey, S.A.: Gaseous protein cations are amphoteric. J. Am. Chem. Soc. 119, 1688-1696 (1997)

15. Williams, E.R.: Proton transfer reactivity of large multiply charged ions. J. Mass Spectrom. 31, 831-842 (1996)

16. Gur, E.H., de Koning, L.J., Nibbering, N.M.M.: The bimolecular gasphase reaction of protonated alkyldipeptides with acetonylacetone. Int. J. Mass Spectrom. Ion Processes 167, 135-147 (1997)

17. Reid, G.E., Tichy, S.E., Perez, J., O’Hair, R.A.J., Simpson, R.J., Kenttämaa, H.I.: N-terminal derivatization and fragmentation of neutral peptides via ion-molecule reactions with acylium ions: 
toward gas-phase Edman degradation? J. Am. Chem. Soc. 123, 1184-1192 (2001)

18. Cook, S.L., Collin, O.L., Jackson, G.P.: Metastable atom-activated dissociation mass spectrometry: leucine/isoleucine differentiation and ring cleavage of proline residues. J. Mass Spectrom. 44, 1211-1223 (2009)

19. Berkout, V.D.: Fragmentation of protonated peptide ions via interaction with metastable atoms. Anal. Chem. 78, 3055-3061 (2006)

20. Misharin, A.S., Silivra, O.A., Kjeldsen, F., Zubarev, R.A.: Dissociation of peptide ions by fast atom bombardment in a quadrupole ion trap. Rapid Commun. Mass Spectrom. 19, 2163 (2005)

21. Berkout, V.D., Doroshenko, V.M.: Fragmentation of phosphorylated and singly charged peptide ions via interaction with metastable ions. Int. J. Mass Spectrom. 278, 150-157 (2008)

22. Gologan, B., Wiseman, J.M., Cooks, R.G.: Ion soft landing: instrumentation, phenomena, and applications. In: Laskin, J., Lifshitz, C. (eds.) Principles of mass spectrometry applied to biomolecules, chapter 12, pp. 443-475. Wiley, Hoboken (2006)

23. Dongre, A.R., Somogyi, A., Wysocki, V.H.: Surface-induced dissociation: an effective tool to probe structure, energetics and fragmentation mechanisms of protonated peptides. J. Mass Spectrom.31, 339-350 (1996)

24. Grill, V., Shen, J., Evans, C., Cooks, R.G.: Collisions of ions with surfaces at chemically relevant energies: instrumentation and phenomena. Rev. Sci. Instrum. 72, 3149-3179 (2001)

25. Wysocki, V.H., Joyce, K.E., Jones, C.M., Beardsley, R.L.: Surface-induced dissociation of small molecules, peptides, and noncovalent protein complexes. J. Am. Soc. Mass Spectrom. 19, 190208 (2008)

26. Beardsley, R.L., Jones, C.M., Galhena, A.S., Wysocki, V.H.: Noncovalent protein tetramers and pentamers with " $n$ " charges yield monomers with n/4 and n/5 charges. Anal. Chem. 81, 1347-1356 (2009)

27. Baer, T., Dunbar, R.C.: Ion spectroscopy: where did it come from; where is it now; and where is it going? J. Am. Soc. Mass Spectrom. 21, 681-693 (2010)

28. Bomse, D.S., Beauchamp, J.L.: Slow multi-photon excitation as a probe of bimolecular and unimolecular reaction energetic-multi-photon dissociation of proton bound alcohol dimers. J. Am. Chem. Soc. 103, 3292-3296 (1981)

29. Little, D.P., Speir, J.P., Senko, M.W., O’Connor, P.B., McLafferty, F. $\mathrm{W}$.: Infrared multiphoton dissociation of large multiply charged ions for biomolecule sequencing. Anal. Chem. 66, 2809-2815 (1994)

30. Brodbelt, J.S., Wilson, J.J.: Infrared multiphoton dissociation in quadrupole ion traps. Mass Spectrom. Rev. 28, 390-424 (2009)

31. Reilly, J.P.: Ultraviolet photofragmentation of biomolecular ions. Mass Spectrom. Rev. 28, 425-447 (2009)

32. Ly, T., Julian, R.R.: Ultraviolet photodissociation: developments towards applications for mass spectrometry-based proteomics. Angew. Chem. Int. Ed. 48, 7130-7137 (2009)

33. McLuckey, S.A., Goeringer, D.E.: Slow heating methods in tandem mass spectrometry. J. Mass Spectrom. 32, 461-474 (1997)

34. Vasicek, L.A., Wilson, J.J., Brodbelt, J.S.: Improved infrared multiphoton dissociation of peptides through N-terminal phosphonite derivatization. J. Am. Soc. Mass Spectrom. 20, 377-384 (2009)

35. Price, W.D., Schnier, P.D., Jockusch, R.A., Strittmatter, E.F., Williams, E.R.: Unimolecular reaction kinetics in the high-pressure limit without collisions. J. Am. Chem. Soc. 118, 10640-10644 (1996)

36. Thompson, M.S., Cui, W.D., Reilly, J.P.: Fragmentation of singly charged peptide ions by photodissociation at $\lambda=157 \mathrm{~nm}$. Angew. Chem. Int. Ed. 43, 4791-4794 (2004)

37. Weber, J.M., Ioffe, I.N., Berndt, K.M., Löffler, D., Friedrich, J., Ehrler, O.T., Danell, A.S., Parks, J.H., Kappes, M.M.: Photoelectron spectroscopy of isolated multiply negatively charged oligonucleotides. $\mathrm{J}$. Am. Chem. Soc. 126, 8585-8589 (2004)

38. Gabelica, G., Tabarin, T., Antoine, R., Rosu, F., Compagnon, I., Broyer, M., De Pauw, E., Dugourd, P.: Electron photodetachment dissociation of DNA polyanions in a quadrupole ion trap mass spectrometer. Anal. Chem. 78, 6564-6572 (2006)

39. Cody, R.B., Freiser, B.S.: Electron impact excitation of ions from organics-alternative to collision-induced dissociation. Anal. Chem. 51, 547-551 (1979)
40. Zubarev, R.A., Kelleher, N.L., McLafferty, F.W.: Electron capture dissociation of multiply charged protein cations. a nonergodic process. J. Am. Chem. Soc. 120, 3265-3266 (1998)

41. Horn, D.M., Ge, Y., McLafferty, F.W.: Activated ion electron capture dissociation for mass spectral sequencing of larger $(42 \mathrm{kDa})$ proteins. Anal. Chem. 72, 4778-4784 (2000)

42. Kjeldsen, F., Budnik, B.A., Haselmann, K.F., Jensen, F., Zubarev, R. A.: Dissociative capture of hot $(3-13 \mathrm{eV})$ electrons by polypeptide polycations: an efficient process accompanied by secondary fragmentation. Chem. Phys. Lett. 356, 201-206 (2002)

43. Zubarev, R.A.: Reactions of polypeptide ions with electrons in the gas phase. Mass Spectrom. Rev. 22, 57-77 (2003)

44. Zubarev, R.A., Budnik, B.A., Nielsen, M.L.: Tandem ionization mass spectrometry of biomolecules. Eur. J. Mass Spectrom. 6, 235-240 (2000)

45. Budnik, B.A., Haselmann, K.F., Zubarev, R.A.: Electron detachment dissociation of peptide di-anions: an electron-hole recombination phenomenon. Chem. Phys. Lett. 342, 299-302 (2001)

46. Nielsen, M.L., Budnik, B.A., Haselmann, K.F., Zubarev, R.A.: Tandem MALDI/EI ionization for tandem Fourier transform ion cyclotron resonance mass spectrometry of polypeptides. Int. J. Mass Spectrom. 226, 181-187 (2003)

47. Song, H., Han, L., Håkansson, K.: Proceedings of the $58^{\text {th }}$ ASMS Conference on Mass Spectrometry and Allied Topics, Salt Lake City, UT (2010)

48. Baba, T., Hashimoto, Y., Hasegawa, H., Hirabayashi, A., Waki, I.: Electron capture dissociation in a radio-frequency ion trap. Anal. Chem. 76, 4263-4266 (2004)

49. McLuckey, S.A., Stephenson Jr., J.L.: Ion/ion chemistry of high-mass multiply charged ions. Mass Spectrom. Rev. 17, 369-407 (1998)

50. Pitteri, S.J., McLuckey, S.A.: Recent developments in the ion/ion chemistry of high-mass multiply charged ions. Mass Spectrom. Rev. 24, 931-958 (2005)

51. McLuckey, S.A., Huang, T.Y.: Ion/ion reactions: new chemistry for analytical mass spectrometry. Anal. Chem. 81, 8669-8676 (2009)

52. McLuckey, S.A.: Biomolecule Ion/Ion Reactions. In: Laskin, J., Lifshitz, C. (eds.) Principles of Mass Spectrometry Applied to Biomolecules, Chapter 14, pp. 519-564. Wiley-Interscience, Hoboken (2006)

53. Stephenson Jr., J.L., McLuckey, S.A.: Ion/Ion reactions in the gasphase: proton transfer reactions involving multiply-charged proteins. $J$. Am. Chem. Soc. 118, 7390-7397 (1996)

54. Scalf, M., Westphall, M.S., Krause, J., Kaufman, S.L., Smith, L.M.: Controlling charge states of large ions. Science 283, 194-197 (1999)

55. Herron, W.J., Goeringer, D.E., McLuckey, S.A.: Gas-phase electron transfer reactions from multiply-charged anions to rare gas cations. $J$. Am. Chem. Soc. 117, 11555-11562 (1995)

56. Syka, J.E.P., Coon, J.J., Schroeder, M.J., Shabanowitz, J., Hunt, D. F.: Peptide and protein sequence analysis by electron transfer dissociation mass spectrometry. Proc. Natl Acad. Sci. USA 101, 9528-9533 (2004)

57. Newton, K.A., McLuckey, S.A.: Gas-phase peptide/protein cationizing agent switching via ion/ion reactions. J. Am. Chem. Soc. 125, 1240412405 (2003)

58. Wells, J.M., Chrisman, P.A., McLuckey, S.A.: Formation and characterization of protein-protein complexes in vacuo. J. Am. Chem. Soc. 125, 7238-7249 (2003)

59. Han, H., McLuckey, S.A.: Selective covalent bond formation in polypeptide ions via gas-phase ion/ion reaction chemistry. J. Am. Chem. Soc. 131, 12884-12885 (2009)

60. Hassell, K.M., Stutzman, J.R., McLuckey, S.A.: Gas phase bio-conjugation of peptides via ion/ion charge inversion: Schiff base formation on the conversion of cations to anions. Anal. Chem. 82, 1594-1597 (2010)

61. He, M., McLuckey, S.A.: Two ion/ion charge inversion steps to form a doubly-protonated peptide from a singly-protonated peptide in the gas phase. J. Am. Chem. Soc. 125, 7756-7757 (2003)

62. He, M., McLuckey, S.A.: Increasing the negative charge of a macroanion in the gas phase via sequential charge inversion reactions. Anal. Chem. 76, 4189-4192 (2004)

63. Coon, J.J., Shabanowitz, J., Hunt, D.F., Syka, J.E.P.: Electron transfer dissociation of peptide anions. J. Am. Soc. Mass Spectrom. 16, 880-882 (2005) 